\title{
Electrical Properties of GaInAsSb/GaSb/GaAlAsSb Double Heterostructure with Low Diameter
}

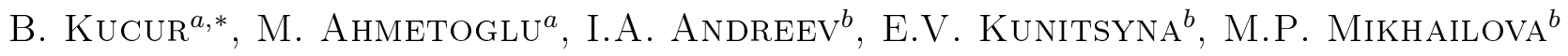 \\ AND Y.P. YAKOVLEV ${ }^{b}$ \\ ${ }^{a}$ Department of Physics, Faculty of Sciences and Arts, Uludag University, 16059 Gorukle, Bursa, Turkey \\ ${ }^{b}$ Ioffe Physical-Technical Institute RAS, Politekhnicheskaya 26, St. Petersburg 194021, Russia
}

$\mathrm{GaInAsSb} / \mathrm{GaSb} / \mathrm{GaAlAsSb}$ double heterostructures are attractive for optoelectronic devices working in the 1.5-4.8 $\mu \mathrm{m}$ wavelength region. In this paper, the current mechanisms of liquid phase epitaxy grown GaInAsSb based double heterostructures with $100 \mu \mathrm{m}$ diameter were investigated in the temperature range 77-350 K. It was found that diffusion current dominates at the high temperature $(>240 \mathrm{~K})$ and small forward bias region, while generation-recombination current dominates at intermediate temperatures $(242-171 \mathrm{~K})$. At low temperature region $(<171 \mathrm{~K})$, the tunneling mechanism of the current flow dominates in both forward and reverse biases.

DOI: $10.12693 /$ APhysPolA.125.411

PACS: 73.40.-c, 78.66. $-\mathrm{w}, 85.60 . \mathrm{Dw}$

\section{Introduction}

GaInAsSb/GaSb/GaAlAsSb heterostructures are of interest due to their importance for promising optoelectronic devices working in the wavelength region $1.5^{-}$ $4.8 \mu \mathrm{m}$. Both efficient light-emitting devices and high-speed detectors have been prepared and may be used for gas pollution monitoring, as well as for optical communications in the new generation of fibers [1-5]. GaSb-based materials are widely used narrow band gap semiconductors for infrared detection in $0.9-2.55 \mu \mathrm{m}$ spectral range. This class of materials has become the subject of extensive investigations because of the progress in developing infrared lasers, light emitting diodes, photodiodes, solar cells, thermophotovoltaic devices for mid-IR spectral range [6-9].

In addition, thermophotovoltaic (TPV) devices based on III-V semiconductor alloys with bandgap energies corresponding to the mid-infrared wavelength range 2.2 to $2.5 \mu \mathrm{m}(0.5$ to $0.56 \mathrm{eV})$ are being developed. To date, the highest performing TPV devices in this wavelength range have been achieved for devices based on GaInAsSb and AlGaAsSb alloys lattice matched to GaSb substrates [10].

In this report, dark current-voltage characteristics of $\mathrm{GaInAsSb} / \mathrm{GaSb} / \mathrm{GaAlAsSb}$ heterostructure with an active area $100 \mu \mathrm{m}$ in diameter, are examined. The results obtained will be useful both for improving the material synthesis technology and for developing optoelectronic devices.

\section{Experimental}

Epitaxial layers of the GaAlAsSb, GaInAsSb solid solutions and the photodiode heterostructures were grown

*corresponding author; e-mail: banukucur@uludag.edu.tr by liquid phase epitaxy (LPE) at Ioffe Physical-Technical Institute RAS. High-quality $\mathrm{Ga}_{0.78} \mathrm{In}_{0.22} \mathrm{As}_{0.18} \mathrm{Sb}_{0.82}$ solid solutions with composition near the miscibility gap boundary were grown at $T=600{ }^{\circ} \mathrm{C}$ on Te-doped GaSb(100) substrates. The lattice mismatch between the GaInAsSb epitaxial layer and substrate which was determined by X-ray diffractometry (XRD) was found to be $\Delta a / a=(2-5) \times 10^{-4}$ at room temperature [11]. The epitaxial layers have shown a good surface morphology and straight interface lines for investigated structures. Mesa samples with a working area $100 \mu \mathrm{m}$ in diameter were fabricated from these structures by photolithography. Thermal evaporated $\mathrm{AuGe} / \mathrm{Ni} / \mathrm{Au}$ and $\mathrm{Cr} / \mathrm{Au} /$ $\mathrm{Ni} / \mathrm{Au}$ ohmic contacts with annealing at $300^{\circ} \mathrm{C}$ for $30 \mathrm{~s}$ in $\mathrm{H}_{2}$ atmosphere were applied to the $p$-GaAlAsSb top of each mesa and to the back of $n$-GaSb substrate, respectively.

Fabricated devices were mounted into a glass dewar with a cold shield for detailed electrical measurements at variable temperatures. $I-V$ characteristics were performed using a Keithley $6517 \mathrm{~A}$ electrometer and $C-V$ measurements were carried out at room temperature with Keithley $590 \mathrm{C}-\mathrm{V}$ analyzer. All measurements were controlled by a computer via an IEEE-488 standard interface so that the data collecting, processing and plotting could be accomplished automatically. Photovoltaic measurements were employed using a $50 \mathrm{~W}$ halogen lamp and THORLABS FES1000 infrared filter. The intensity of halogen lamp radiation was varied by changing the current through it. The intensity of the incident light was measured by THORLABS photodiode amplifier PDA200C with BPW41N silicon PIN photodiode.

\section{Results and discussion}

High level of dark current is one of the basic problems of photodiodes [12]. It is important to understand the nature of dark current mechanisms of the structure. 
Figure 1 shows current-voltage $(I-V)$ characteristics of the $\mathrm{GaInAsSb} / \mathrm{GaSb} / \mathrm{GaAlAsSb}$ structure with an active area $100 \mu \mathrm{m}$ in diameter.

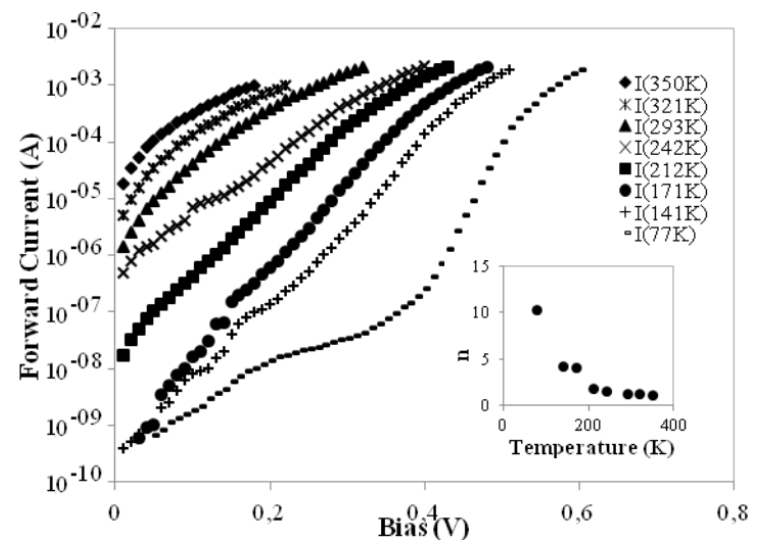

Fig. 1. Forward $I-V$ characteristics of the structure at several temperatures, in the inset ideality factor values.

The dependence of forward current on voltage can be described by this empirical expression [13]:

$$
I=I_{0}\left[\exp \left(\frac{q V}{n k T}\right)-1\right],
$$

where $n$ is the ideality factor which was calculated from slope of the linear region of the forward bias $\ln I-V$ (in semi-log scale) plot and can be written as

$$
n=\frac{q}{k T}\left(\frac{\mathrm{d} V}{\mathrm{~d}(\ln I)}\right) \text {. }
$$

It is seen from the inset of Fig. 1 that the $n$ value increases with decreasing temperature from $n=1.12 \pm 0.03$ at the temperature of $350 \mathrm{~K}$ to $n=10.3$ at $T=77 \mathrm{~K}$. At high temperatures $(T \geq 293 \mathrm{~K})$ and temperatures between $242 \mathrm{~K}$ and $212 \mathrm{~K}$ the forward current was determined by two different mechanisms of the flow of the current, by diffusion and recombination mechanisms, respectively. At the low temperatures $(T \leq 200 \mathrm{~K})$ the contribution of tunneling component was carried in essential to the mechanism of the flow of the current. This was proved by the weak dependence of the forward current on temperature at this temperature region.

Under forward bias the flow of holes from the $p$-region reaches the negative contact unimpeded, but electrons encounter a high barrier at the heterointerface and find themselves in a deep potential well. Some electrons and holes with high energy pass over the barriers at the heterointerface. However, the carrier recombination at the heterointerface, with subsequent tunneling of carriers through it, makes a substantial contribution to the total current. At low temperatures, the tunneling processes become dominant, since passing over barriers becomes improbable. This is confirmed by the $n$ values obtained $(n \geq 2)$ for low temperature region.

Figure 2 shows dependences of the dark current on reverse bias at several temperatures. It is seen that, at room temperature, a quadratic dependence of the current on the bias is observed for small biases $(V \leq 0.4 \mathrm{~V})$ that indicates the predominance of the generative character of the current.

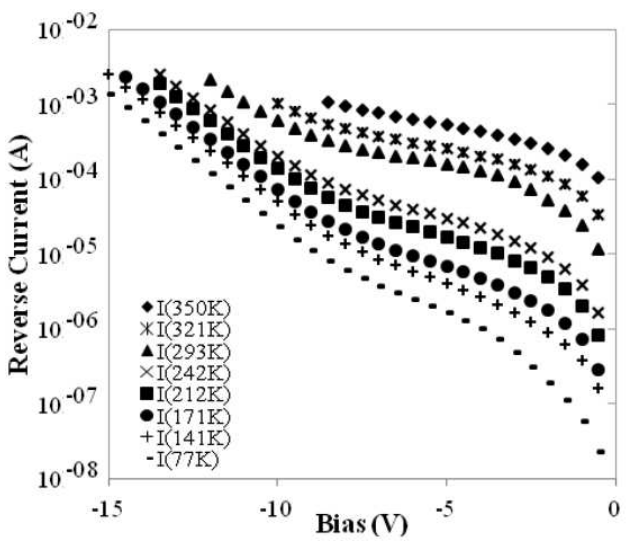

Fig. 2. Reverse current-voltage characteristics of the structure at several temperatures.

Figure 3 shows the reverse current versus $10^{3} / T$ as a function of reverse bias. The experimental data agree with the dependence for the generation-recombination (GR) current. This kind of current has a temperature dependence of the form $I \propto T^{3 / 2} \exp \left(-E_{\mathrm{g}} / 2 k T\right)$. The activation energy, determined by this dependence at the temperatures over the range from $293 \mathrm{~K}$ to $350 \mathrm{~K}$ is $E_{\mathrm{a}}=0.29 \mathrm{eV}$ which is close to half band gap value of narrow gap structure. The deviation from the calculated dependence for the GR current is due to tunneling over whole reverse bias range at low temperatures.

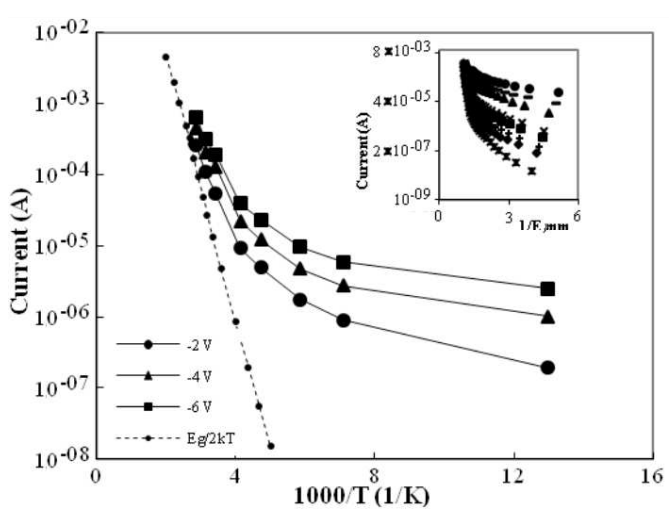

Fig. 3. Reverse current as a function of reciprocal temperature at different reverse biases. In the inset, reverse biased dark current dependence on maximum electric field at several temperatures.

To further test the tunneling model, the $I-V$ characteristics of the investigated samples were used to obtain the dependence of current on $1 / E_{\mathrm{m}}$ at fixed $E_{\mathrm{g}}$, as shown in Fig. 3. Let us note that the tunneling current for direct gap semiconductors is strongly dependent on the ratio $E_{\mathrm{g}}^{3 / 2} / E_{\mathrm{m}}$, therefore, this mechanism can account for 
the nearly exponential dependence of dark current on applied voltage. The maximum junction electric field can be found experimentally by $E_{\mathrm{m}}=\left[2 q N_{\mathrm{d}}\left(V_{\mathrm{bi}}-V\right) / \varepsilon_{2}\right]^{1 / 2}$. The data show considerable curvature at low electric fields, with the curvature more pronounced at higher temperatures. This behavior indicates that tunneling becomes the dominant source of leakage with increasing field and decreasing temperature.

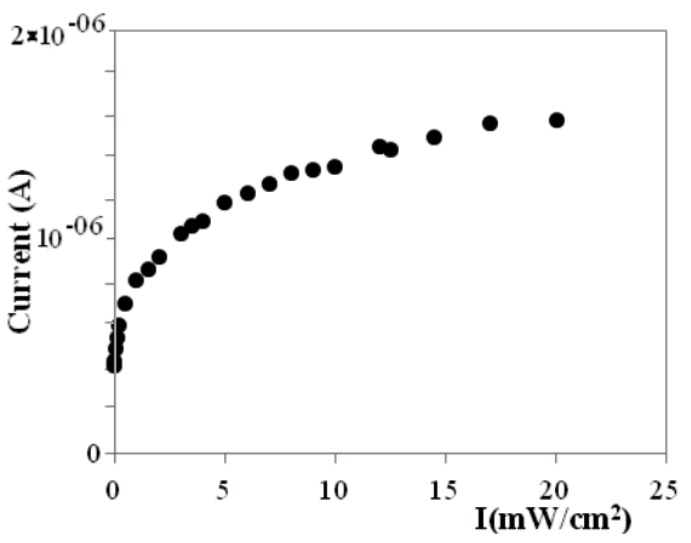

Fig. 4. Dependence of short-circuit current on the light intensity.

In the photovoltaic regime energy characteristics are the dependences either of short-circuit current or open circuit voltage on the intensity of incident light. Figure 4 shows variation of the short-circuit current with light intensity for the photodiode. As seen from them, with the high intensity of the incident light the law of variation in these dependences substantially differs from linear. For short-circuit current, the appearance of this nonlinearity is connected with the increase a voltage drop across the serial resistance of the base.

\section{Conclusion}

In this paper we have presented the dark current characteristics of GaInAsSb/GaSb/GaAlAsSb heterostructure with low diameter active area in the temperature range of $77 \mathrm{~K}$ to $350 \mathrm{~K}$. The investigation of the nature of dark current has shown that diffusion current dominates at high temperatures in small forward bias region. On the other hand, reverse dark current consists of generation-recombination and tunneling components. Studies of these heterojunctions provided the physical basis for the fabrication of the photodetectors operating in the wavelength range 1.6-2.2 $\mu \mathrm{m}$, important for third-generation infrared fiber-optic communication systems and suitable for tasks involving ecology and protection of the environment.

\section{References}

[1] A.N. Baranov, N. Bertu, Y. Cumminal, G. Boissier, C. Alibert, A. Joullie, Appl. Phys. Lett. 71, 735 (1997).

[2] J.R. Meyer, C.A. Hoffman, F.J. Bartoli, L.R. RamMohan, Appl. Phys. Lett. 67, 757 (1995).

[3] A.N. Baranov, D.E. Dzhurtanov, A.N. Imenkov, A.A. Rogachev, Yu.M. Shernyakov, Yu.P. Yakovlev, Sov. Phys. Semicond. 20, 1385 (1986).

[4] I.A. Andreev, M.A. Afrailov, A.N. Baranov, S.G. Konnikov, M.A. Mirsagatov, M.P. Mikhailova, O.V. Salata, V.E. Umansky, G.M. Filaretova, Yu.P. Yakovlev, Sov. Phys. Tech. Lett. 15, 253 (1989).

[5] M.A. Afrailov, A.N. Baranov, A.P. Dmitriev, M.P. Mikhailova, Yu.P. Smorchkova, I.N. Timchenko, V. Sherstnev, Yu. Yakovlev, I.N. Yassievich, Sov. Phys. Semicond. 24, 8 (1990).

[6] M.H.M. Reddy, J.T. Olesberg, C. Cao, J.P. Prineas, Semicond. Sci. Technol. 21, 267 (2006).

[7] A.M. Monakhov, V.V. Sherstnev, A.P. Astakhova, Yu.P. Yakovlev, G. Boissier, R. Teissier, A.N. Baranov, Appl. Phys. Lett. 94, 051102 (2009).

[8] M.P. Mikhailova, I.A. Andreev, E.V. Kunitsyna, Yu.P. Yakovlev, Proc. SPIE 7355, 735511 (2009).

[9] M.G. Mauk, V.M. Andreev, Semicond. Sci. Technol. 18, 191 (2003).

[10] E.V. Kunitsyna, T.V. L'vova, M.S. Dunaevskii, Ya.V. Terent'ev, A.N. Semenov, V.A. Solov'ev, B.Ya. Meltser, S.V. Ivanov, Yu.P. Yakovlev, Appl. Surf. Sci. 256, 18 (2010).

[11] I.A. Andreev, E.V. Kunitsyna, M.P. Mikhailova, Yu.P. Yakovlev, Mater. Res. Soc. Symp. Proc. 744, 589 (2003).

[12] I.A. Andreev, M.A. Afrailov, A.N. Baranov, V.G. Danil'chenko, M.A. Mirsagatov, M.P. Mikhailova, Yu.P. Yakovlev, Tech. Phys. Lett. 12, 542 (1986).

[13] S. Sze, Physics of Semiconductor Devices, Wiley, New York 1981. 